\title{
MicroRNA-613 inhibits proliferation and invasion of renal cell carcinoma cells through targeting FZD7
}

\author{
HAITAO SONG ${ }^{1,2}$, YONGHAO NAN ${ }^{1}$, XINSHENG WANG ${ }^{1}$, GANG ZHANG ${ }^{1}$, SHI ZONG ${ }^{1}$ and XIANGBO KONG \\ ${ }^{1}$ Department of Urinary Surgery, China-Japan Union Hospital, Jilin University, Changchun, Jilin 130033; \\ ${ }^{2}$ Department of Critical Care Medicine, Jilin Province People's Hospital, Changchun, Jilin 130021, P.R. China
}

Received September 7, 2016; Accepted May 18, 2017

DOI: $10.3892 / \mathrm{mmr} .2017 .7076$

\begin{abstract}
MicroRNAs (miRNAs) have emerged as critical regulators in cancer progression. miR-613 has been reported as a tumor suppressor gene in many types of human cancers. However, the function of miR-613 in renal cell carcinoma (RCC) remains unclear. In the present study, the authors aimed to detect the expression of miR-613 and its function in RCC cell lines. miR-613 was reported to be significantly downregulated RCC cell lines. Functional analyses demonstrated that overexpression of miR-613 significantly decreased RCC cell proliferation and invasion. Bioinformatics analysis showed that Frizzled7 (FZD7) was a predicted target of miR-613, which was verified by dual-luciferase reporter assay, reverse transcription quantitative-polymerase chain reaction and western blot analysis. Restoration of FZD7 significantly reversed the suppressive effects of miR-613 on RCC cell proliferation and invasion. Taken together, the results of the present study indicated that miR-613 functions as a tumor suppressor that inhibits RCC cell proliferation and invasion by targeting and inhibiting FZD7, providing novel insight into RCC pathogenesis and a potential therapeutic target for RCC.
\end{abstract}

\section{Introduction}

Renal cell carcinoma (RCC) is the most prevalent malignancy of the adult kidney, accounting for $\sim 90 \%$ of kidney tumors and $3 \%$ of cancers in adults $(1,2)$. The morbidity and mortality of RCC have increased steadily in recent years (3). Despite advances in cancer therapies, RCC is an intractable disease,

Correspondence to: Dr Xiangbo Kong, Department of Urinary Surgery, China-Japan Union Hospital, Jilin University, 126 Xiantai Street, Changchun, Jilin 130033, P.R. China

E-mail: kongxiangbo_cju@163.com

Abbreviations: miRNAs, microRNAs; RCC, renal cell carcinoma; FZD7, Frizzled7; 3'-UTR, 3'-untranslated region; RT-qPCR, reverse transcription-quantitative polymerase chain reaction; FBS, fetal bovine serum

Key words: FZD7, microRNA-613, renal cell carcinoma, cell proliferation, cell invasion and most affected patients undergo metastasis development and relapse after treatment $(4,5)$. The molecular mechanism of RCC pathogenesis is still elusive, hampering the development of effective therapeutics. Therefore, specifically targeted therapies to improve the survival rate of RCC based on molecular mechanisms are urgently required.

MicroRNAs (miRNAs) are a class of conserved non-coding RNAs of 22 nucleotides in length that serve an important role in regulating gene expression $(6,7)$. miRNAs can directly target the 3'-untranslated region (UTR) of the mRNA to induce mRNA degradation and translation inhibition $(6,7)$. A growing body of evidence has indicated that miRNAs are critical regulators of cancer development and progression that regulate cell proliferation, apoptosis, differentiation, migration and invasion by functioning as either oncogenes or tumor suppressors (8-10). Increasing evidence has reported that various miRNAs are dysregulated in RCC, contributing to the pathogenesis of RCC and can serve as potential diagnostic and prognostic biomarkers and therapeutic targets of RCC (11-13). However, the precise role of miRNAs in RCC remains largely unknown.

Frizzled7 (FZD7) is an important co-receptor in the Wnt signaling pathway $(14,15)$. Aberrant expression of FZD7 occurs frequently in numerous cancers and is associated with abnormal activation of the Wnt signaling pathway (16-19). FZD7 regulates cancer cell proliferation, invasion and metastasis by promoting the Wnt signaling pathway $(20,21)$. Wnt signaling is extensively involved in the progression of RCC (22). High expression of FZD7 has been reported in RCC tissues and cell lines and contributes to Wntmediated RCC progression (23). Therapy targeting the FZD7 signaling pathway may have potential therapeutic value in the treatment of RCC.

Two recent studies indicated that miR-613 is a tumorassociated miRNA in various cancer types $(24,25)$; however, the role of miR-613 in RCC remains unknown. The present study intended to investigate the expression and role of miR-613 in RCC. The results demonstrated that miR-613 was frequently decreased in RCC cell lines. Overexpression of miR-613 significantly inhibited RCC cell proliferation and invasion. Bioinformatics analysis and dual-luciferase reporter assay demonstrated that FZD7 was a direct target of miR-613. Further data indicated that FZD7 contributed to miR-613-endowed effects, including cell proliferation and 
invasion. Taken together, these findings indicated that miR-613 inhibits RCC cell proliferation and invasion by targeting FZD7, suggesting a potential therapeutic candidate for RCC.

\section{Materials and methods}

Cell lines. Human RCC cell lines, including ACHN, 786-O, A498 and Caki-2, and a human renal proximal tubule epithelial cell line (HK-2) were purchased from the American Type Culture Collection (Manassas, VA, USA). RCC cells were cultured in Dulbecco's modified Eagle medium (Invitrogen; Thermo Fisher Scientific, Inc., Waltham, MA, USA) supplemented with $1 \%$ penicillin/streptomycin mix (Sigma-Aldrich; Merck KGaA, Darmstadt, Germany) and $10 \%$ fetal bovine serum (FBS; Invitrogen; Thermo Fisher Scientific, Inc.). HK-2 cells were cultured with F-12K medium (Gibco; Thermo Fisher Scientific, Inc.) containing 10\% FBS (Invitrogen; Thermo Fisher Scientific, Inc.) and 1\% penicillin/streptomycin mix (Sigma-Aldrich; Merck KGaA). All cells were cultured within a humidified atmosphere containing $5 \% \mathrm{CO}_{2} / 95 \%$ air at $37^{\circ} \mathrm{C}$.

$R N A$ extraction and reverse transcription-quantitative polymerase chain reaction ( $R T-q P C R)$ analysis. Total RNA was extracted from tissues or cells using TRIzol reagent (Invitrogen; Thermo Fisher Scientific, Inc.). Reverse transcription of mRNA and miRNA were performed by Super M-MLV Reverse Transcriptase (BioTeke Corporation, Beijing, China) or miScript reverse transcription kit (Qiagen $\mathrm{GmbH}$, Hilden, Germany), respectively, according to the manufacturer's instructions. PCR amplification was carried out with an Applied Biosystems AB7500 Real Time PCR system (Applied Biosystems; Thermo Fisher Scientific, Inc.) under the following conditions: $94^{\circ} \mathrm{C}$ for $10 \mathrm{~min} ; 35$ cycles of $94^{\circ} \mathrm{C}$ for $20 \mathrm{sec}, 55^{\circ} \mathrm{C}$ for $30 \mathrm{sec}$ and $72^{\circ} \mathrm{C}$ for $30 \mathrm{sec}$; and $72^{\circ} \mathrm{C}$ for $5 \mathrm{~min}$. The reactions were performed using sing Power SYBR-Green PCR Master Mix (Applied Biosystems; Thermo Fisher Scientific, Inc.). The primers used were as follows: miR-613 forward, 3'-CGTTTC TTCCTTGTAAGGA-5' and reverse, 5'-CCCAAGCTTGTT GGAGAACAGCAGCGAGGAC-3'; U6 forward, 5'-GCTTCG GCAGCACATATACTAAAAT-3' and reverse, 5'-CGCTTC ACGAATTTGCGTGTCAT-3'; FZD7, forward: 5'-CCAACG GCCTGATGTACTTT-3' and reverse, 5'-ATGAAGTAGCAG CCCGACAG-3'; and GAPDH forward, 5'-CCATGTTCGTCA TGGGTGTG-3' and reverse, 5'-GGTGCTAAGCAGTTGGTG GTG-3'. The data was analyzed by the $2^{-\triangle \Delta C q}$ method (26). The relative expression of FZD7 was obtained by normalization to GAPDH. The relative expression of miR-613 was obtained by normalization to U6.

Cell transfection. The miR-613 mimics and negative control miRNAs (miR-NC) used in the current study were synthesized by Shanghai GenePharma Co., Ltd. (Shanghai, China). The full-length cDNA of FZD7 without 3'-UTR region was cloned into the pcDNA3.0 vector (Invitrogen; Thermo Fisher Scientific, Inc.) to generate pcDNA3/FZD7 overexpressing vectors. The miRNAs and vectors were transiently transfected into cells using Lipofectamine 2000 (Invitrogen; Thermo Fisher Scientific, Inc.) according to the manufacturer's instructions.
MTT assay. Cells were plated into 96-well plates at a density of $1 \times 10^{4}$ cells/well and cultured for $24 \mathrm{~h}$. The cells were then transfected with miR-613 mimics or miR-NC for 24, 48 and $72 \mathrm{~h}$. Thereafter, $20 \mu 15 \mathrm{mg} / \mathrm{ml}$ MTT solution (Sigma-Aldrich; Merck KGaA) was added to each well. Following incubation for another $4 \mathrm{~h}$, the medium was discarded and $150 \mu \mathrm{l}$ dimethyl sulfoxide (Sigma-Aldrich; Merck KGaA) was added to each well to dissolve the formazan products. The absorbance of the converted dye was detected at a wavelength of $490 \mathrm{~nm}$ using a microplate spectrophotometer (Bio-Tek Instruments, Inc., Winooski, VT, USA).

Colony formation assay. Cells were transfected with miR-613 mimics or miR-NC for $48 \mathrm{~h}$, and then the transfected cells were seeded into six-well plates at a density of 100 cells/well and cultured in growth medium containing $0.3 \%$ noble agar for 14 days. The colonies were stained with $0.1 \%$ crystal violet (Sigma-Aldrich; Merck KGaA) and counted under the microscope (Olympus Corporation, Tokyo, Japan).

Cell invasion assay. The cell invasion assay was performed by Matrigel invasion assay. Briefly, Transwell membrane filter inserts were precoated with Matrigel (BD Biosciences, Franklin Lakes, NJ, USA). A total of $500 \mu 1$ serum-free medium containing $1 \times 10^{5}$ cells transfected with miR-613 mimics or miR-NC was placed into the top chamber. Meanwhile, $500 \mu \mathrm{l}$ growth medium containing $10 \%$ FBS was placed into the lower chamber. Cells were cultured at $37^{\circ} \mathrm{C}$ for $24 \mathrm{~h}$, noninvasive cells on the top well were gently scraped by a cotton swab. The invaded cells were fixed with methanol and stained with $0.1 \%$ crystal violet (Sigma-Aldrich; Merck KGaA). Stained cells were counted under light microscopy (Olympus Corporation).

Western blot analysis. Cells were harvested and lysed in radioimmunoprecipitation assay buffer (Beyotime Institute of Biotechnology, Haimen, China). Protein concentration was measured using a bicinchoninic acid assay kit (Beyotime Institute of Biotechnology). A total of $25 \mu \mathrm{g}$ protein was separated by SDS-PAGE (Sangon Biotech Co., Ltd., Shanghai, China). The separated proteins were electro-transferred to a polyvinylidene difluoride membrane (EMD Millipore, Billerica, MA, USA). The membrane was blocked in $3 \%$ nonfat milk and incubated with primary anti-FZD7 (1:250; Abcam, Cambridge, UK; cat. no. ab64636) or anti-GAPDH (1:1,000; Abcam; cat. no. ab9485) antibodies at $4^{\circ} \mathrm{C}$ overnight, followed by incubation with horseradish peroxidase-conjugated secondary antibodies (1:2,000; BIOSS, Beijing, China; cat. no. bs-0295G-HRP) for $1 \mathrm{~h}$ at $37^{\circ} \mathrm{C}$. The protein bands were developed using an enhanced chemiluminescence western blotting kit (EMD Millipore). Densitometry analysis of the protein blots was conducted using Image-Pro Plus 6.0 software (Media Cybernetics, Inc., Rockville, MD, USA) and normalized to GAPDH.

Dual-luciferase reporter assay. The 3'-UTR of FZD7 containing miR-613 binding sites was cloned into the pmirGLO Dual-Luciferase Vector (Promega Corporation, Madison, WI, USA) to generate wild-type (WT) pmirGLO-FZD7. The 3'-UTR of FZD7 containing mutations in the miR-613 recognition sites was synthesized using a Site-Directed 
Mutagenesis kit (Agilent Technologies, Inc., Santa Clara, CA, USA) and cloned into the pmirGLO Dual-Luciferase Vector to generate mutant (MT) pmirGLO-FZD7. The pmirGLO Dual-Luciferase Vectors were cotransfected with miR-613 mimics or miR-NC using Lipofectamine 2000 (Invitrogen; Thermo Fisher Scientific, Inc.) and incubated for $48 \mathrm{~h}$. Relative luciferase activity was detected using the Dual-Glo Luciferase Assay system (Promega Corporation).

Data analysis. All results were presented as means \pm standard deviation and analyzed using SPSS software (version, 11.5; SPSS Inc., Chicago, IL, USA). Differences were assessed for significance by Student's t-test and one-way analysis of variance followed by Bonferroni's post hoc test according to the data characteristics. $\mathrm{P}<0.05$ was considered to indicate a statistically significant difference.

\section{Results}

Downregulation of miR-613 occurs frequently in RCC. To explore the relevance of miR-613 in RCC, the authors examined the expression profile of miR-613 in four RCC cell lines (ACHN, 786-O, A498 and Caki-2) using the human renal proximal tubule epithelial cell line HK-2 as a control. The results demonstrated that the expression of miR-613 was markedly decreased by $80 \%$ in $\mathrm{ACHN}(\mathrm{P}<0.05), 77 \%$ in $786-\mathrm{O}(\mathrm{P}<0.05)$, $61 \%$ in $\mathrm{A} 498(\mathrm{P}<0.05)$ and $56 \%$ in Caki-2 $(\mathrm{P}<0.05)$ cells as compared with HK-2 cells (Fig. 1). These results indicated that downregulation of miR-613 is a common event in RCC that may contribute to the pathogenesis of RCC.

Overexpression of miR-613 inhibits RCC cell proliferation. To investigate the possible biological function of miR-613 in RCC cells, the authors transfected ACHN and 786-O cells with miR-613 mimics or negative miRNAs (miR-NC). The transfection efficiency was verified by RT-qPCR analysis, which indicated that the miR-613 expression level was significantly increased by 6.87 -fold $(\mathrm{P}<0.01)$ in miR-613 mimic-transfected ACHN cells (Fig. 2A) and increased by 5.96 -fold $(\mathrm{P}<0.01)$ in miR-613 mimic-transfected 786-O cells (Fig. 2B) compared with the control. Following this, the effect of miR-613 overexpression on RCC cells was examined by MTT assay. The results identified that overexpression of miR-613 significantly decreased RCC proliferation of ACHN (Fig. 2C; $\mathrm{P}<0.05$ ) and 786-O (Fig. 2D; $\mathrm{P}<0.05$ ) cells. To confirm the inhibitory effect of miR-613 on RCC cell proliferation, the authors further performed a colony formation assay. The results demonstrated that miR-613 overexpression significantly inhibited colony-forming capacity by $40 \%$ in ACHN (Fig. $2 \mathrm{E}$; P<0.05) and 62.5 in 786-O (Fig. 2F; $\mathrm{P}<0.05$ ) cells. These results indicated that miR-613 suppresses RCC cell proliferation.

Overexpression of miR-613 suppresses RCC cell invasion. To further investigate the biological role of miR-613 in RCC cells, the authors performed an invasion assay to detect the effect of miR-613 overexpression on RCC cell invasion. The results demonstrated that miR-613 overexpression significantly repressed the invasive ability of ACHN by $55 \%$ (Fig. 3A; $\mathrm{P}<0.05$ ) and $786-\mathrm{O}$ by $61 \%$ (Fig. $3 \mathrm{~B} ; \mathrm{P}<0.05$ ) cells. These results suggested that miR-613 inhibits RCC cell invasion.

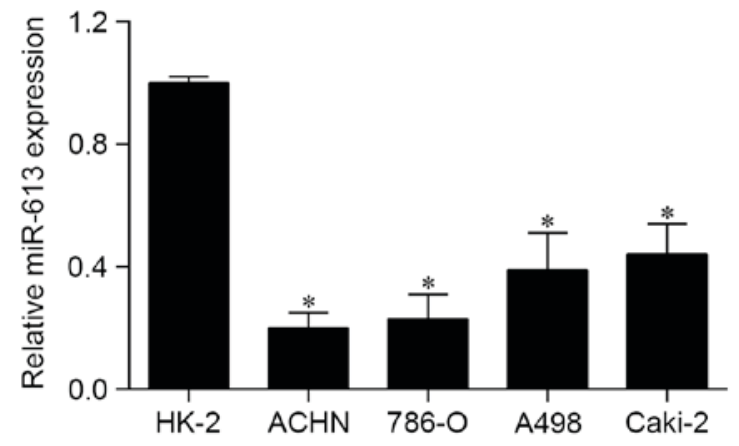

Figure 1. The expression of miR-613 was decreased in RCC cell lines. The miR-613 expression in RCC cell lines (ACHN, 786-O, A498 and Caki-2) and human renal proximal tubule epithelial cell line HK-2 was detected by reverse transcription-quantitative polymerase chain reaction analysis. The data are presented as means \pm standard deviation. ${ }^{*} \mathrm{P}<0.05$ vs. HK-2. miR, microRNA; RCC, renal cell carcinoma.

FZD7 is the direct target of miR-613. To investigate the molecular mechanism by which miR-613 inhibits RCC cell proliferation and invasion, potential target genes of miR-613 were screened by bioinformatics analysis. Interestingly, the authors reported that FZD7, a well-known oncogene in various cancers (18), was predicted to be a target of miR-613 (Fig. 4A). To verify whether FZD7 is the direct target gene of miR-613, a dual-luciferase reporter assay was conducted. Cotransfection of the WT FZD7 3'-UTR construct with miR-613 mimics inhibited luciferase activity by $58 \%$ in ACHN (Fig. 4B; $\mathrm{P}<0.05$ ) and $62 \%$ in $786-\mathrm{O}$ (Fig. 4C; $\mathrm{P}<0.05)$ cells. However, overexpression of miR-613 reported no obvious effect on the MT FZD7 3'-UTR construct (Fig. 4B and $C ; P>0.05$ ). Furthermore, the effect of mIR-613 on FZD7 expression was detected. The results demonstrated that miR-613 overexpression inhibited FZD7 mRNA expression by $56 \%$ in $\mathrm{ACHN}$ (Fig. $5 \mathrm{~A} ; \mathrm{P}<0.05$ ) and $53 \%$ in $786-\mathrm{O}$ (Fig. 5B; P<0.05) cells. Furthermore, miR-613 overexpression remarkably suppressed FZD7 protein expression by $81 \%$ in ACHN (Fig. 5C; $\mathrm{P}<0.01$ ) and $89 \%$ in 786-O (Fig. 5D; P<0.01) cells. Taken together, these results indicated that miR-613 directly targets the 3'-UTR of FZD7 and inhibits FZD expression in RCC cells.

Restoration of FZD7 rescues the tumor suppressive effect of $m i R-613$. To investigate whether the tumor suppressive effect of miR-613 on RCC cell proliferation and invasion was mediated by FZD7 repression, FZD7 expression in miR-613-overexpressing cells was restored by transfection with pcDNA3 vectors expressing FZD7 without the 3'-UTR region. The results indicated that the reduced expression of FZD7 induced by miR-613 mimics was significantly restored by $92 \%$ in ACHN (Fig. 6A; $\mathrm{P}<0.01$ ) and 83\% in 786-O (Fig. 6B; P<0.01) cells transfected with pcDNA3/FZD7 vector. As expected, the inhibitory effect of miR-613 on RCC cell proliferation was reversed by $90 \%$ in ACHN and $87 \%$ in 786-O (Fig. 7A; $\mathrm{P}<0.05)$ cells by FZD7 overexpression. In addition, the inhibitory effect of miR-613 on RCC cell invasion was reversed by $81 \%$ in $\mathrm{ACHN}$ and $77 \%$ in $786-\mathrm{O}$ (Fig. 7A; $\mathrm{P}<0.05$ ) cells by FZD7 overexpression. These results implied that miR-613 inhibits RCC cell proliferation and invasion by targeting FZD7. 

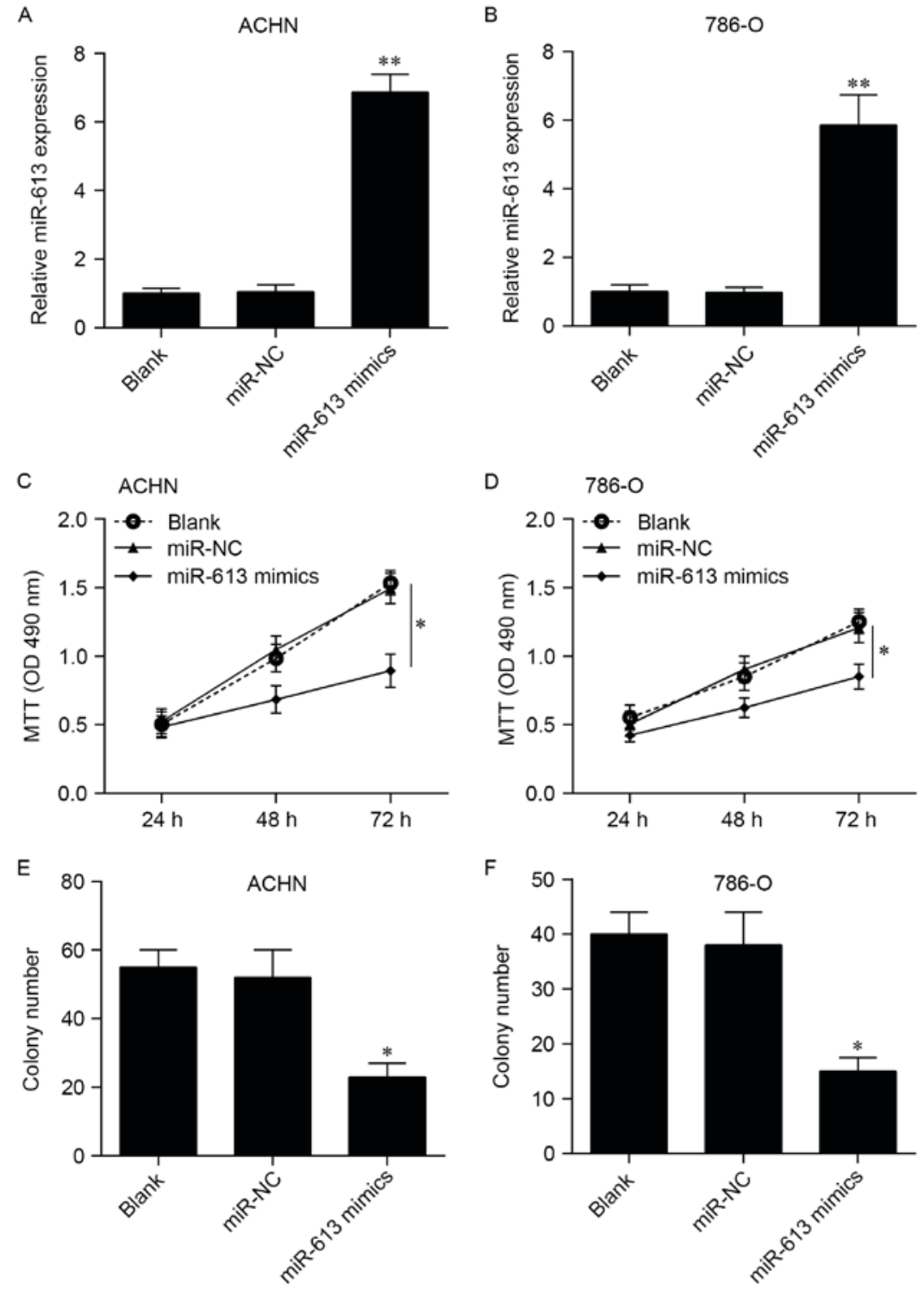

Figure 2. miR-613 inhibited renal cell carcinoma cell proliferation. ACHN and 786-O cells were transiently transfected with miR-613 mimics and miR-NC. Blank, cells were without treatment. The expression of miR-613 in (A) ACHN and (B) 786-O cells was detected by reverse transcription-quantitative polymerase chain reaction following $48 \mathrm{~h}$ of transfection. ${ }^{* *} \mathrm{P}<0.01$ vs. blank and miR-NC. MTT assay of (C) ACHN and (D) 786-O cells following transfection for 24, 48 and $72 \mathrm{~h}$. "P<0.05 as indicated. The colony-forming capacity of (E) ACHN and (F) 786-O cells was detected by colony formation assay following incubation for 14 days. The colonies were stained with crystal violet and counted under the microscope. The data are presented as means \pm standard deviation. ${ }^{*} \mathrm{P}<0.05$ vs. blank and miR-NC. miR, microRNA; NC, negative control.

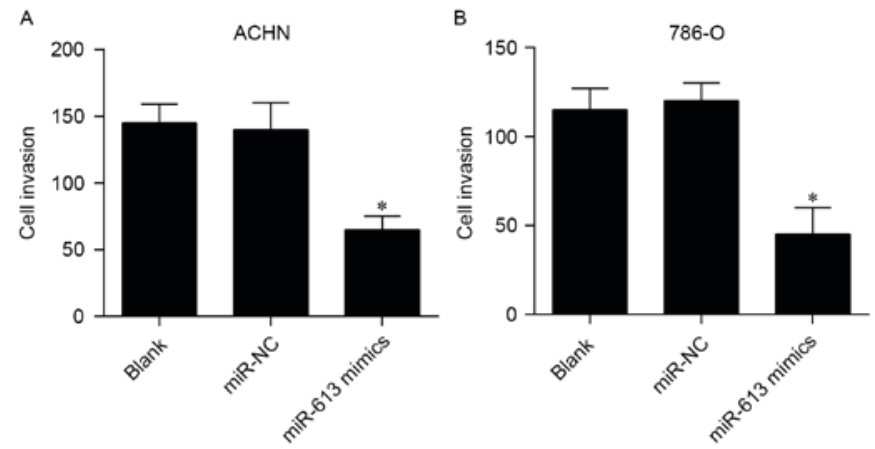

Figure 3. miR-613 repressed renal cell carcinoma cell invasion. Cell invasion of (A) ACHN and (B) 786-O cells was detected by Transwell invasion assay. Cells transfected with miR-613 mimics or miR-NC were cultured at $37^{\circ} \mathrm{C}$ for $24 \mathrm{~h}$ in the Transwell assay. The data are presented as means \pm standard deviation. ${ }^{*} \mathrm{P}<0.05$ vs. blank and miR-NC. miR, microRNA; NC, negative control.

\section{Discussion}

A growing body of evidence suggests that miRNAs regulate a variety of genes involved in RCC progression (11-13). Therefore, identification of RCC-associated miRNAs as biomarkers for diagnosis, prognosis and therapeutic targets for RCC treatment is of great importance. In the present study, the authors have revealed that miR-613 is frequently downregulated RCC cell lines. Functional analysis demonstrated that miR-613 overexpression repressed RCC cell proliferation and invasion. Moreover, the findings identified and verified FZD7 as the direct target gene of miR-613 in RCC cells. Rescuing FZD7 expression significantly reversed the suppressive effect of miR-613 on RCC proliferation and invasion. Taken together, the results of the current study suggested that miR-613 inhibits RCC cell proliferation and invasion through targeting FZD7. 
A

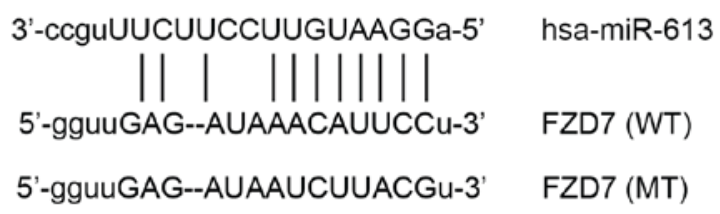

B

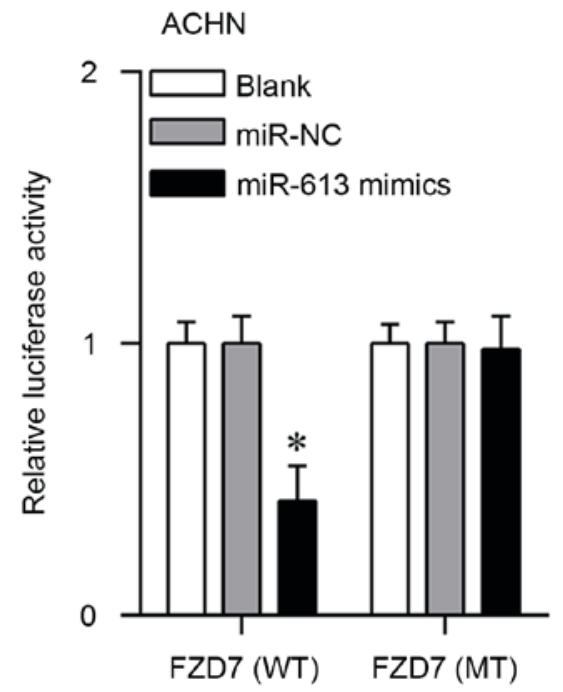

C

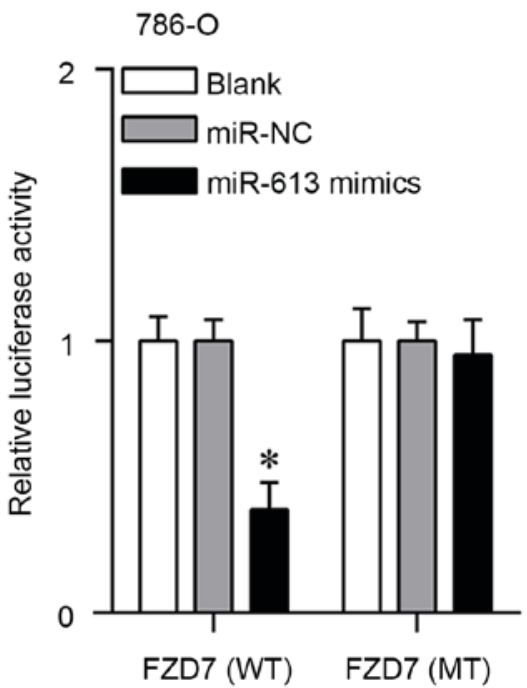

Figure 4. miR-613 targeted the 3'-UTR of FZD7. (A) A schematic diagram representing the miR-613-binding sites within the 3'-UTR of FZD7. The dual-luciferase assay in (B) ACHN and (C) 786-O cells transfected with miR-613 mimics and FZD7 3'-UTR constructs. Following 48 h of incubation, the relative luciferase activity was detected using the dual-Glo luciferase assay system. The data are presented as means \pm standard deviation. "P<0.05 vs. blank and miR-NC. miR, microRNA; UTR, untranslated region.

A

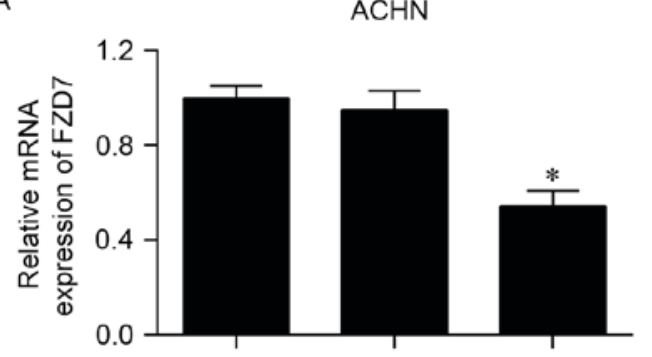

C

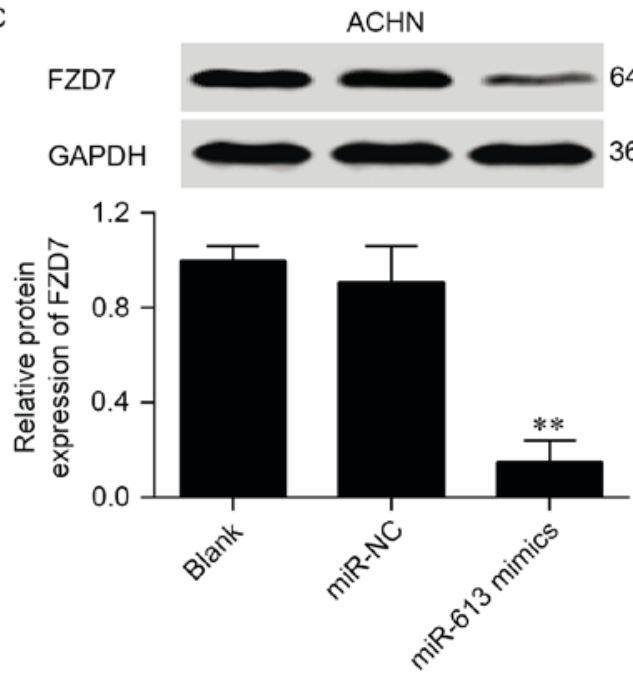

B

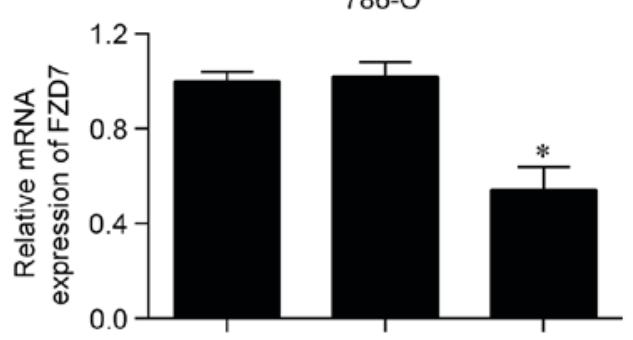

D
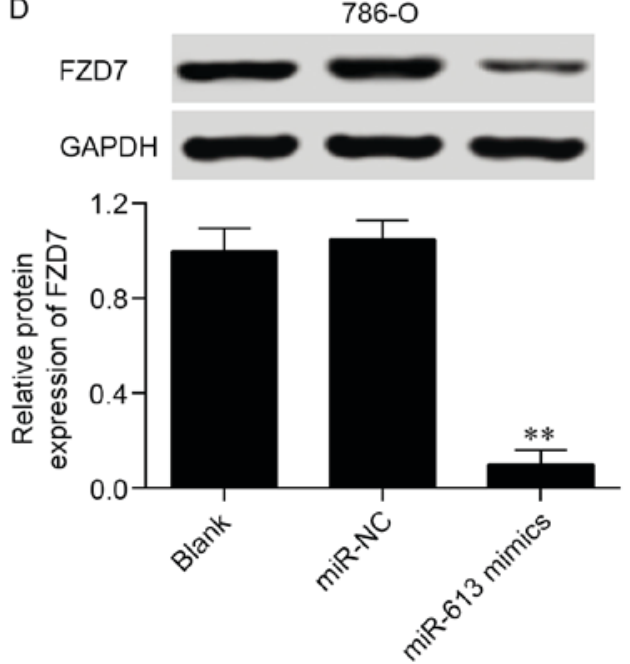

Figure 5. miR-613 repressed FZD7 expression. ACHN and 786-O cells were transfected with miR-617 mimics or miR-NC for $48 \mathrm{~h}$ and harvested for analysis. The mRNA expression of FZD7 in (A) ACHN and (B) 786-O cells was examined by reverse transcription-quantitative polymerase chain reaction analysis. The protein expression of FZD7 in (C) ACHN and (D) 786-O cells was detected by western blot analysis. Relative protein expression was analyzed using Image-Pro Plus 6.0 software. The data are presented as means \pm standard deviation. ${ }^{*} \mathrm{P}<0.05,{ }^{* *} \mathrm{P}<0.01$ vs. blank and miR-NC. miR, microRNA; NC, negative control. 

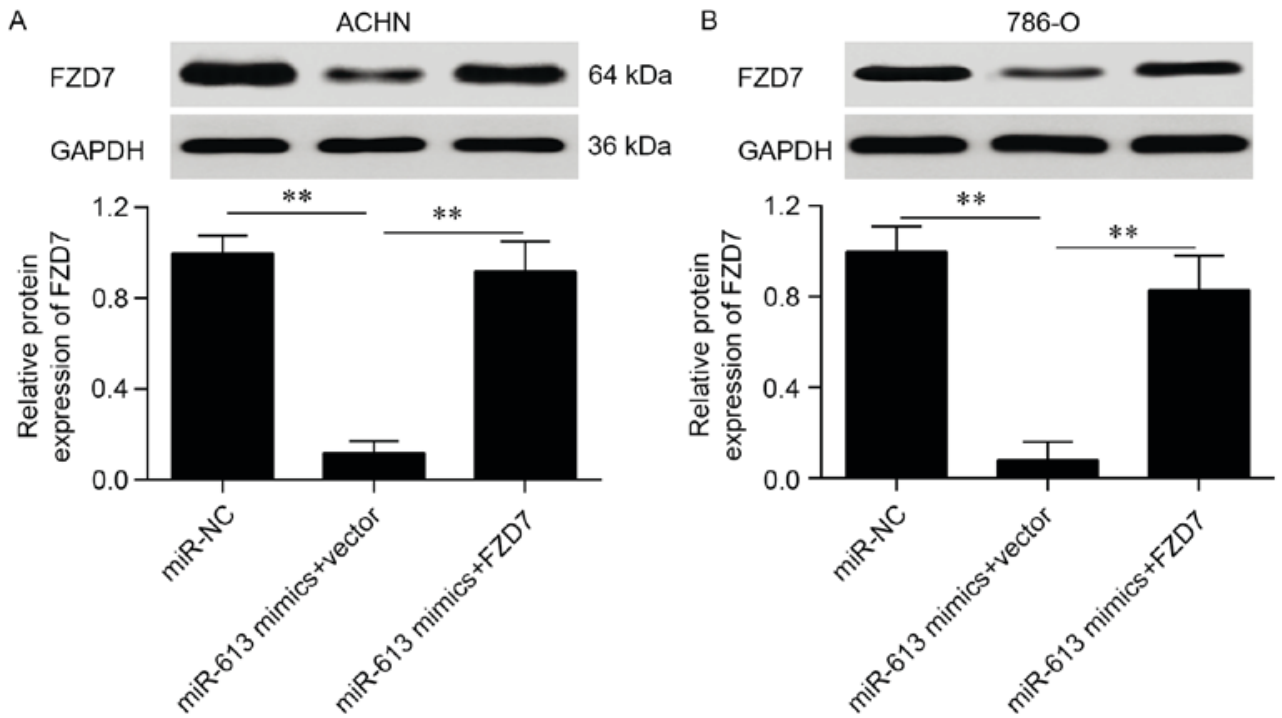

Figure 6. Restoration of FZD7 expression. ACHN and 786-O cells were cotransfected with miR-613 mimics and the pcDNA3/FZD7 expression vector without the 3'-untranslated region for $48 \mathrm{~h}$. Vector, empty vector used as control; miR-613 mimics + vector, cells were transfected with miR-613 mimics and empty vector; miR-613 mimics + FZD7, cells were transfected with miR-613 mimics and pcDNA3/AEG-1 expression vector. Protein expression of FZD7 in (A) ACHN and (B) 786-O cells was detected by western blot analysis. Relative protein expression was analyzed using Image-Pro Plus 6.0 software. The data are presented as means \pm standard deviation. ${ }^{* *} \mathrm{P}<0.01$ as indicated. miR, microRNA.

A

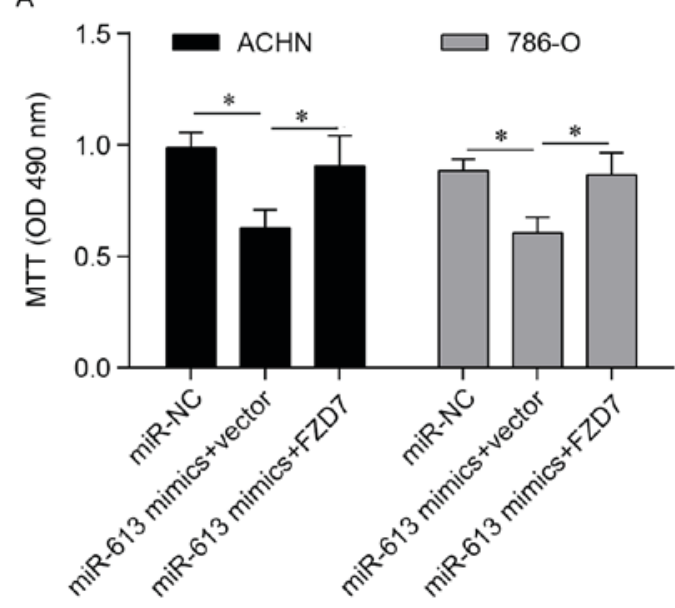

B

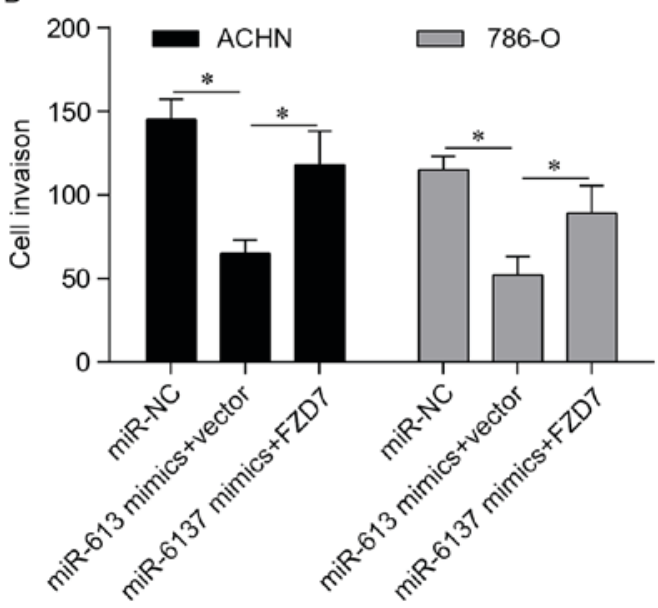

Figure 7. Overexpression of FZD7 restored the inhibitory effect of miR-613 on RCC cell proliferation and invasion. ACHN and 786-O cells were cotransfected with miR-613 mimics and pcDNA3/FZD7 expression vector without the 3'-untranslated region. RCC cell (A) proliferation and (B) invasion were detected by MTT and invasion assay, respectively. The data are presented as means \pm standard deviation. ${ }^{~} \mathrm{P}<0.05$ as indicated. miR, microRNA; RCC, renal cell carcinoma; OD, optical density.

Two recent studies have reported that several miRNAs are abnormally expressed in RCC and involved in RCC pathogenesis. For example, miR-196a and miR-149-5p are significantly downregulated in RCC and associated with proliferation, apoptosis, and migration $(27,28)$. Das et al (29) reported that overexpression of miR-214 inhibited RCC proliferation by targeting the insulin-like growth factor-1 receptor. Gao et al (30) reported that miR-155 promotes RCC proliferation and invasion by inhibiting E2F2. In the current study, the authors stated that miR-613 also functioned as an RCC-associated miRNA. However, the precise role of miR-613 in RCC remains unknown. miR-613 was downregulated in RCC cell lines and overexpression of miR-613 inhibited RCC cell proliferation and invasion. These data suggested that miR-613 functions as a tumor suppressor in RCC.

To date, various studies have proposed a tumor-suppressive role of miR-613 in many types of cancers. Decreased expression of miR-613 has been suggested as novel diagnostic and prognostic biomarker for esophageal squamous-cell carcinoma (31) and ovarian cancer (32). Fu et al (33) reported that miR-613 suppressed ovarian cancer cell proliferation and invasion through targeting of KRAS. In non-small-cell lung cancer, miR-613 is significantly decreased, and overexpression of miR-613 induces cell cycle arrest and inhibits cell growth through inhibition of CDK4 (34). Qiu et al (25) reported that miR-613 suppressed papillary thyroid cancer cell growth, migration and invasion via regulation of sphingosine kinase 2 . 
A more recent study (24) reveals that miR-613 represses breast cancer cell proliferation and invasion by targeting vascular endothelial growth factor A. These studies indicated a tumor suppressive role of miR-613 that functions through inhibition of various oncogenes. Consistent with these studies, these findings supported the notion that miR-613 is a tumor suppressor that inhibits RCC cell proliferation and invasion by targeting FZD7, a well-known oncogene (18). Interestingly, these findings are in line with a recent study demonstrating that miR-613 suppresses prostate cancer cell proliferation and invasion by targeting and inhibiting FZD7 (35).

FZD7 is the Wnt receptor most commonly increased in numerous cancers that serves an important role in cancer development and progression (18). Overexpression of FZD7 is also identified in RCC associated with RCC cell proliferation (23). Therapies targeted at FZD7, including siRNA knockdown, neutralizing antibody and the extracellular peptide of FZD7, show considerable anti-tumor activity in vitro and in vivo $(15,19,21,36,37)$. In recent years, miRNAs have emerged as novel tools for gene expression. Therefore, targeting FZD7 by specific miRNAs may have the potential to treat cancers. To date, several miRNAs, including miR-27a (38), miR-27b (39), miR-142-3p (40) and miR-199a (41), have been indicated to inhibit cancer progression through targeting of FZD7. In addition, other miRNAs, such as miR-1 and miR-184, also target and inhibit FZD7 in other pathological processes $(42,43)$. These studies raise the possibility that targeting FZD7 by specific miRNAs is practicable. In the current study, the authors identified that miR-613 could also target and regulate FZD7 expression, as confirmed by dual-luciferase reporter assay, RT-qPCR and western blot analysis. The present findings are consistent with a recent study demonstrating that miR-613 targets and inhibits FZD7 (35), further confirming the targeted relationship between miR-613 and FZD7.

In conclusion, the data presented by the present study suggested that miR-613 may serve as an anti-cancer therapeutic for RCC. Overexpression of miR-613 in RCC cell lines resulted in suppression of cell proliferation and invasion by inhibiting FZD7. The study may help to further elucidate the molecular mechanisms underlying RCC pathogenesis and provide candidate therapeutic targets for RCC.

\section{References}

1. Ljungberg B, Campbell SC, Choi HY, Jacqmin D, Lee JE, Weikert $S$ and Kiemeney LA: The epidemiology of renal cell carcinoma. Eur Urol 60: 615-621, 2011.

2. Bex A, Jonasch E, Kirkali Z, Mejean A, Mulders P, Oudard S, Patard JJ, Powles T, van Poppel H and Wood CG: Integrating surgery with targeted therapies for renal cell carcinoma: Current evidence and ongoing trials. Eur Urol 58: 819-828, 2010.

3. Siegel RL, Miller KD and Jemal A: Cancer statistics, 2016. CA Cancer J Clin 66: 7-30, 2016.

4. Motzer RJ and Molina AM: Targeting renal cell carcinoma. J Clin Oncol 27: 3274-3276, 2009.

5. Albiges L, Choueiri T, Escudier B, Galsky M, George D, Hofmann F, Lam T, Motzer R, Mulders P, Porta C, et al: A systematic review of sequencing and combinations of systemic therapy in metastatic renal cancer. Eur Urol 67: 100-110, 2015.

6. Bartel DP: MicroRNAs: Genomics, biogenesis, mechanism, and function. Cell 116: 281-297, 2004.

7. Winter J, Jung S, Keller S, Gregory RI and Diederichs S: Many roads to maturity: microRNA biogenesis pathways and their regulation. Nat Cell Biol 11: 228-234, 2009.
8. Manikandan J, Aarthi JJ, Kumar SD and Pushparaj PN: Oncomirs: The potential role of non-coding microRNAs in understanding cancer. Bioinformation 2: 330-334, 2008.

9. Pang JC, Kwok WK, Chen Z and Ng HK: Oncogenic role of microRNAs in brain tumors. Acta Neuropathol 117: 599-611, 2009.

10. Silber J, James CD and Hodgson JG: microRNAs in gliomas: Small regulators of a big problem. Neuromolecular Med 11: 208-222, 2009.

11. Gu L, Li H, Chen L, Ma X, Gao Y, Li X, Zhang Y, Fan Y and Zhang X: MicroRNAs as prognostic molecular signatures in renal cell carcinoma: A systematic review and meta-analysis. Oncotarget 6: 32545-32560, 2015.

12. Grange C, Collino F, Tapparo M and Camussi G: Oncogenic micro-RNAs and renal cell carcinoma. Front Oncol 4: 49, 2014.

13. Li M, Wang Y, Song Y, Bu R, Yin B, Fei X, Guo Q and Wu B: MicroRNAs in renal cell carcinoma: A systematic review of clinical implications (Review). Oncol Rep 33: 1571-1578, 2015.

14. Abu-Elmagd M, Garcia-Morales C and Wheeler GN: Frizzled7 mediates canonical Wnt signaling in neural crest induction. Dev Biol 298: 285-298, 2006.

15. Asad M, Wong MK, Tan TZ, Choolani M, Low J, Mori S, Virshup D, Thiery JP and Huang RY: FZD7 drives in vitro aggressiveness in Stem-A subtype of ovarian cancer via regulation of non-canonical Wnt/PCP pathway. Cell Death Dis 5: e1346, 2014.

16. Reya $\mathrm{T}$ and Clevers $\mathrm{H}$ : Wnt signalling in stem cells and cancer. Nature 434: 843-850, 2005

17. Klaus $\mathrm{A}$ and Birchmeier W: Wnt signalling and its impact on development and cancer. Nat Rev Cancer 8: 387-398, 2008.

18. King TD, Zhang W, Suto MJ and Li Y: Frizzled7 as an emerging target for cancer therapy. Cell Signal 24: 846-851, 2012.

19. Yang L, Wu X, Wang Y, Zhang K, Wu J, Yuan YC, Deng X, Chen L, Kim CC, Lau S, et al: FZD7 has a critical role in cell proliferation in triple negative breast cancer. Oncogene 30: 4437-4446, 2011.

20. Deng B, Zhang Y, Zhang S, Wen F, Miao Y and Guo K: MicroRNA-142-3p inhibits cell proliferation and invasion of cervical cancer cells by targeting FZD7. Tumour Biol 36: 8065-8073, 2015.

21. Deng B, Zhang S, Miao Y, Zhang Y, Wen F and Guo K: Down-regulation of Frizzled-7 expression inhibits migration, invasion, and epithelial-mesenchymal transition of cervical cancer cell lines. Med Oncol 32: 102, 2015.

22. Xu Q, Krause M, Samoylenko A and Vainio S: Wnt signaling in renal cell carcinoma. Cancers (Basel) 8: pii: E57, 2016.

23. Xu R, Zeng S, Xie W, Sun C, Chen YL, Chen MJ and Zhang L: The expression and function of Frizzled-7 in human renal cell carcinoma. Clin Transl Oncol 18: 269-276, 2016.

24. Wu J, Yuan P, Mao Q, Lu P, Xie T, Yang H and Wang C: miR-613 inhibits proliferation and invasion of breast cancer cell via VEGFA. Biochem Biophys Res Commun 478: 274-278, 2016.

25. Qiu W, Yang Z, Fan Y and Zheng Q: MicroRNA-613 inhibits cell growth, migration and invasion of papillary thyroid carcinoma by regulating SphK2. Oncotarget 7: 39907-39915, 2016.

26. Livak KJ and Schmittgen TD: Analysis of relative gene expression data using real-time quantitative PCR and the 2(-Delta Delta C(T)) Method. Methods 25: 402-408, 2001.

27. Li Y, Jin L, Chen D, Liu J, Su Z, Yang S, Gui Y, Mao X, Nie G and Lai Y: Tumor suppressive miR-196a is associated with cellular migration, proliferation and apoptosis in renal cell carcinoma. Mol Med Rep 14: 560-566, 2016.

28. Jin L, Li Y, Liu J, Yang S, Gui Y, Mao X, Nie G and Lai Y: Tumor suppressor miR-149-5p is associated with cellular migration, proliferation and apoptosis in renal cell carcinoma. Mol Med Rep 13: 5386-5392, 2016.

29. Das F, Dey N, Bera A, Kasinath BS, Ghosh-Choudhury N and Choudhury GG: MicroRNA-214 reduces insulin-like growth factor-1 (IGF-1) receptor expression and downstream mTORC1 signaling in renal carcinoma cells. J Biol Chem 291: 14662-14676, 2016.

30. Gao Y, Ma X, Yao Y, Li H, Fan Y, Zhang Y, Zhao C, Wang L, Ma M, Lei $Z$ and Zhang $X$ : miR-155 regulates the proliferation and invasion of clear cell renal cell carcinoma cells by targeting E2F2. Oncotarget 7: 20324-20337, 2016.

31. Guan S, Wang C, Chen X, Liu B, Tan B, Liu F, Wang D, Han L, Wang L, Huang X, et al: miR-613: A novel diagnostic and prognostic biomarker for patients with esophageal squamous cell carcinoma. Tumour Biol 37: 4383-4391, 2016. 
32. Zhang $\mathrm{X}$ and Zhang H: Diminished miR-613 expression as a novel prognostic biomarker for human ovarian cancer. Eur Rev Med Pharmacol Sci 20: 837-841, 2016.

33. Fu X, Cui Y, Yang S, Xu Y and Zhang Z: MicroRNA-613 inhibited ovarian cancer cell proliferation and invasion by regulating KRAS. Tumour Biol 37: 6477-6483, 2016.

34. Li D, Li DQ, Liu D and Tang XJ: miR-613 induces cell cycle arrest by targeting CDK4 in non-small cell lung cancer. Cell Oncol (Dordr) 39: 139-147, 2016

35. Ren W, Li C, Duan W, Du S, Yang F, Zhou J and Xing J: MicroRNA-613 represses prostate cancer cell proliferation and invasion through targeting Frizzled7. Biochem Biophys Res Commun 469: 633-638, 2016.

36. Pode-Shakked N, Harari-Steinberg O, Haberman-Ziv Y, Rom-Gross E, Bahar S, Omer D, Metsuyanim S, Buzhor E, Jacob-Hirsch J, Goldstein RS, et al: Resistance or sensitivity of Wilms' tumor to anti-FZD7 antibody highlights the Wnt pathway as a possible therapeutic target. Oncogene 30: 1664-1680, 2011.

37. Wei W, Chua MS, Grepper S and So SK: Soluble Frizzled-7 receptor inhibits Wnt signaling and sensitizes hepatocellular carcinoma cells towards doxorubicin. Mol Cancer 10: 16, 2011.

38. Chen Z, Ma T, Huang C, Zhang L, Lv X, Xu T, Hu T and Li J: miR-27a modulates the MDR1/P-glycoprotein expression by inhibiting FZD7/ $\beta$-catenin pathway in hepatocellular carcinoma cells. Cell Signal 25: 2693-2701, 2013.
39. Geng Y, Lu X, Wu X, Xue L, Wang X and Xu J: MicroRNA-27b suppresses Helicobacter pylori-induced gastric tumorigenesis through negatively regulating Frizzled7. Oncol Rep 35: 2441-2450, 2016

40. Deng B, Zhang Y, Zhang S, Wen F, Miao Y and Guo K: MicroRNA-142-3p inhibits cell proliferation and invasion of cervical cancer cells by targeting FZD7. Tumour Biol 36: 8065-8073, 2015.

41. Song J, Gao L, Yang G, Tang S, Xie H, Wang Y, Wang J, Zhang Y, Jin J, Gou Y, et al: miR-199a regulates cell proliferation and survival by targeting FZD7. PLoS One 9: e110074, 2014.

42. Takahashi Y, Chen Q, Rajala RV and Ma JX: MicroRNA-184 modulates canonical Wnt signaling through the regulation of frizzled-7 expression in the retina with ischemia-induced neovascularization. FEBS Lett 589: 1143-1149, 2015.

43. Lu TY, Lin B, Li Y, Arora A, Han L, Cui C, Coronnello C, Sheng Y, Benos PV and Yang L: Overexpression of microRNA-1 promotes cardiomyocyte commitment from human cardiovascular progenitors via suppressing WNT and FGF signaling pathways. J Mol Cell Cardiol 63: 146-154, 2013. 